The Journal of Engineering and Exact Sciences - jCEC, Vol. 06 N. 03 (2020)

journal homepage: https://periodicos.ufv.br/ojs/jcec

doi: 10.18540/jcecvl6iss3pp0221-0227

OPEN ACCESS - ISSN: 2527-1075

\title{
ANÁLISE DO DIÂMETRO E DA VELOCIDADE DE BOLHAS DE AR SOB DIFERENTES TENSÕES SUPERFICIAIS: ESTUDO EXPERIMENTAL E SIMULAÇÃO NUMÉRICA CFD
}

\section{ANALYSIS OF DIAMETER AND VELOCITY OF AIR BUBBLES UNDER DIFFERENT SURFACE TENSIONS: EXPERIMENTAL AND CFD STUDY}

\author{
H. H. JACOMINI ${ }^{1}$, J. N. M. BATISTA ${ }^{1}$ e R. BÉTTEGA ${ }^{1, *}$ \\ ${ }^{1}$ Universidade Federal de São Carlos, Departamento de Engenharia Química
}

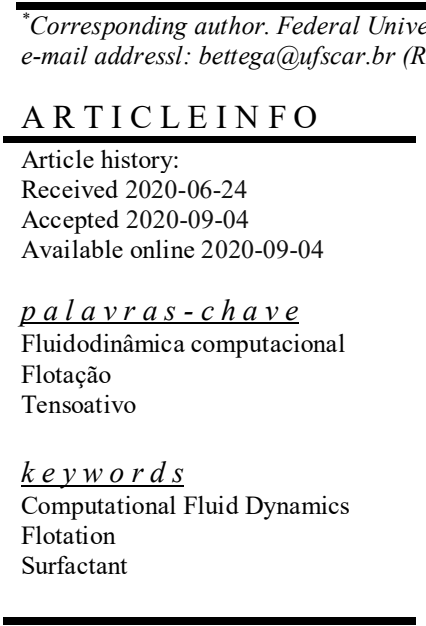

\begin{abstract}
A B S T R A C T
In this work, the air bubbles generation in a flotation tank was evaluated using different surface tensions through experimental tests and by computational fluid dynamics (CFD). The simulations were conducted using the FLUENT® 18.2 version software. To verify the model, the bubble velocity and the bubble diameter simulated data were compared to the experimental results, quantitatively and qualitatively. Through the experiments and by the CFD simulations, it was possible to infer the behavior and dynamics of the bubbles for the different surface tensions, with acceptable deviations between the bubble velocity data obtained experimentally and through simulations. The experimental and simulated diameters of bubbles followed the same trend.
\end{abstract}

\section{R E S UM O}

No presente trabalho, avaliou-se a formação de bolhas de ar em um tanque de flotação empregando diferentes tensões superficiais através de testes experimentais e utilizando a fluidodinâmica computacional (Computational Fluid Dynamics - CFD). As simulações foram conduzidas utilizando o software FLUENT® 18.2. Para a verificação do modelo proposto, os dados simulados de velocidade e diâmetro de bolha foram comparados aos resultados experimentais, tanto quantitativamente quanto qualitativamente. Através dos experimentos e das simulações CFD foi possível obter o comportamento e a dinâmica das bolhas para as diferentes tensões superficiais, verificando-se desvios aceitáveis entre os dados de velocidade de bolha obtidos experimentalmente e por meio de simulações. Os diametros das bolhas experimentais e simulados seguiram a mesma tendência. 
NOM EN C L A T UR A

Fs: força de tensão superficial por unidade de volume $\left[\mathrm{N} / \mathrm{m}^{3}\right]$

$\vec{g}$ : aceleração da gravidade $\left[\mathrm{m} / \mathrm{s}^{2}\right]$

$\hat{n}$ : superfície unitária do vetor normal [-]

$P$ : pressão $[\mathrm{Pa}]$

$t$ : tempo [s]

$\vec{u}$ : vetor velocidade $[\mathrm{m} / \mathrm{s}]$

$x$ : coordenada cartesiana $[\mathrm{m}]$

\section{Letras gregas}

$\alpha$ : fração volumétrica [-]

$\Theta$ : diâmetro do orifício [m]

к: curvatura da superfície local [-]

$\mu$ : viscosidade [Pa.s]

$\rho:$ massa específica $\left[\mathrm{kg} / \mathrm{m}^{3}\right]$

$\sigma:$ coeficiente de tensão superficial [-]

$\mu$ : viscosidade [Pa.s]

\section{Subscritos}

$g$ : fase gasosa

$l$ : fase líquida

\section{INTRODUÇ̃̃O}

A Flotação é um método utilizado para separar e remover impurezas que se encontram em suspensão na solução, através da introdução de bolhas de ar. As espécies em suspensão se aderem às bolhas de ar formando agregados pelas partículas hidrofóbicas e, por serem menos densas que o fluido, dirigem-se à superfície (Rodrigues, 2004).

O papel das bolhas de ar é o de coletar e transportar as partículas suspensas até a superfície, onde há a formação da espuma, da qual se faz a separação das partículas. Por isso, é de extrema importância a utilização de sistemas de aeração que possibilitem o controle do tamanho e da uniformidade das bolhas, juntamente com a utilização de tensoativos, para que se possa ter uma melhor separação através da flotação (Penna et al., 2003).

Uma variável física muito importante para o método de flotação é o diâmetro das bolhas, visto que estas influenciam diretamente na eficiência da separação da mistura e no holdup de ar. Quando o gás é introduzido no sistema ocorre o deslocamento do nível da fase líquida. Com isso, a fração volumétrica deslocada, ou seja, a fração de gás na mistura gáslíquido é chamada de holdup. Assim, o arranjo do tamanho de partículas demanda de uma faixa de tamanho de bolhas que maximize a captura de partículas por parte das bolhas, fazendo com que o processo de flotação seja otimizado (Rodrigues,
2004; Reis Filho et al., 2014).

O tamanho das bolhas liberadas na mistura é controlado pelo ajuste da vazão e da pressão de emissão de ar. Bolhas com diâmetros pequenos têm uma área superficial grande, facilitando (em comparação a bolhas de volumes superiores) a maior coleta de partículas suspensas com base no mesmo volume de ar. Isso é algo de grande interesse, pois, assim, o processo de flotação é mais eficiente, tendo em vista com um menor volume de bolha a colisão bolha-partícula é melhor. Por outro lado, as bolhas de tamanho diminuto apresentam uma velocidade de ascensão baixa podendo ser inferior à velocidade descendente da polpa, o que pode levar a perdas de partículas hidrofóbicas no fluxo de material não flotado (Penna et al., 2003).

De uma forma geral, a velocidade de escoamento do ar e da fase líquida são aspectos fluidodinâmicos que também apresentam influência significativa no processo, pois afetam diretamente a distribuição e o formato das bolhas. $\mathrm{O}$ tamanho das bolhas é quase que, exclusivamente, determinado pelo método de produção das mesmas e pelas propriedades do sistema que as contém, tais como a tensão superficial, a densidade e a viscosidade (Abbassi et al., 2017).

A tensão superficial é resultado de um desequilíbrio de forças existente entre as moléculas que se encontram na superfície em relação às encontradas no interior de um líquido. Enquanto as moléculas localizadas no interior da solução apresentam um elevado número de interações intermoleculares com as moléculas pelas quais é circundada, aquelas na interfase líquido-ar apresentam um número menor de interações (Behring et al., 2004). Tal desequilíbrio de forças gera uma força resultante que atrai as moléculas da superfície para o interior do líquido, causando um considerável obstáculo para a formação de bolhas, gotas e a nucleação de cristais em líquidos. Essas forças de coesão tendem a diminuir a área superficial ocupada pelo líquido, forçando gotas a adotarem a forma esférica. Dá-se o nome de tensão superficial a esse tipo de força, a qual é geralmente quantificada a partir do trabalho necessário para aumentar a área superficial. Os tensoativos são substâncias capazes de diminuir a tensão superficial de uma substância na qual esteja dissolvido. Quando em solução, os tensoativos, preferencialmente, ocupam a superfície do líquido, diminuindo as forças de coesão existentes e, consequentemente, a tensão superficial. Cada tensoativo possui uma concentração micelar crítica (CMC), sendo específicos para cada processo industrial (Shawn et al., 1975).

É de extrema importância que se conheça o comportamento das bolhas de ar em água em diferentes misturas, além de suas características, visto que as bolhas são responsáveis por fazer o transporte das partículas em suspensão da mistura/solução até a superfície. Assim, a aplicação de simulação numérica computacional pode contribuir para o melhor entendimento do processo em questão.

Através da fluidodinâmica computacional, Abbassi et al. (2017), avaliaram a formação e o descolamento de bolhas de ar em água e em soluções de glicerina sob diferentes condições operacionais. Adicionalmente, Abbassi et al. (2018) 
estudaram a dinâmica de uma única bolha de ar, a velocidade instantânea da fase líquida ao redor da bolha e a coalescência de duas bolhas em líquidos de diferentes viscosidades. Ambos os estudos foram conduzidos empregando o VOF (Volume of Fluid). Rodrigues e Béttega (2018) analisaram o escoamento bifásico (água-ar) em uma unidade de Flotação por $\mathrm{Ar}$ Dissolvido (DAF). Os autores avaliaram diferentes abordagens para os modelos multifásicos, de turbulência e de arraste, bem como diferentes condições de contorno para modelar a superfície do tanque DAF. Posteriormente, Rodrigues et al. (2019) aplicaram a fluidodinâmica computacional e as equações de balanço populacional em simulações bifásicas (água-ar) do processo de flotação por ar dissolvido (DAF).

Dada à importância do processo de flotação, o objetivo do trabalho consistiu em avaliar o efeito do tensoativo na formação das bolhas, analisando o diâmetro e velocidade das bolhas. $\mathrm{O}$ estudo foi conduzido através de testes experimentais e por meio da fluidodinâmica computacional, empregando a versão estudante do software FLUENT® 18.2 .

\section{METODOLOGIA EXPERIMENTAL}

O aparato experimental empregado no trabalho é mostrado na Figura 1. A unidade é constituída por recipientes de acrílico de dimensão $12,5 \times 12,5 \times 13 \mathrm{~cm}$, bomba de ar elétrica e rotâmetro. Os recipientes foram preenchidos com diferentes proporções de água e etanol (tensoativo). O diâmetro do orifício de injeção de ar é igual a $0,2 \mathrm{~mm}$. A vazão de alimentação de ar foi mantida a $27,1 \mathrm{~mL} / \mathrm{min}$. Os valores de tensão superficial para $0,20,40,60,80$ e $100 \%$ de etanol em volume foram de $0,082,0,058,0,056,0,055,0,053$ e 0,049 $\mathrm{N} / \mathrm{m}$, respectivamente. A tensão superficial foi obtida pelo método "Peso da gota", o qual faz uso da Lei de Tate.

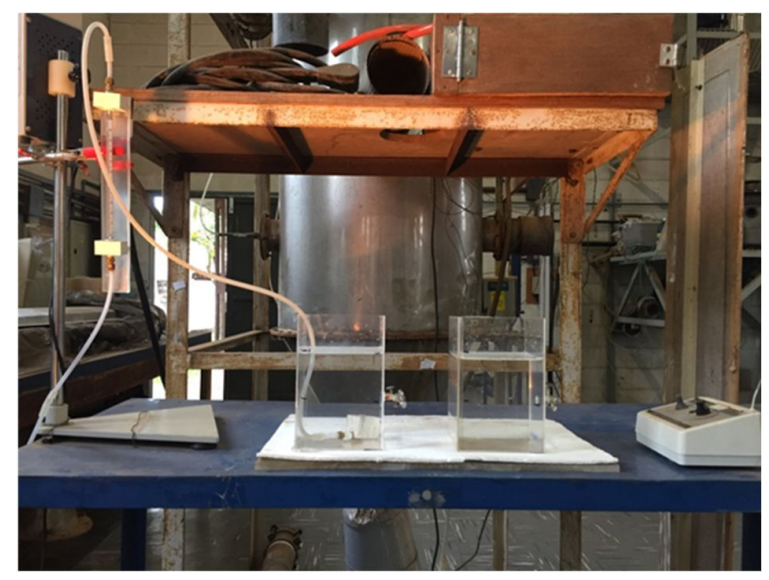

Figura 1 - Aparato experimental.

Para medir a velocidade e diâmetro das bolhas de ar para as diferentes tensões superficiais, os experimentos foram filmados empregando uma câmera de alta velocidade e os dados analisados no software do software Redlake.

\section{METODOLOGIA MATEMÁTICA}

\subsection{Modelagem Matemática}

O modelo VOF (Volume of Fluid) pode modelar dois ou mais fluidos imiscíveis, uma única equação de momentum é resolvida e o campo de velocidade resultante é compartilhado entre as fases e a fração de volume de cada um dos fluidos é rastreada em todo o domínio computacional. Este método apresenta vantagens de fácil compreensão, baixa complexidade computacional e alta precisão (Abbassi et al., 2017). O método VOF é aplicado para capturar a interface, a fração volumétrica $\alpha$ de cada fase $k$ em uma célula computacional é rastreada em todo o domínio.

A equação da continuidade é mostrada na Equação (1).

$$
\nabla \cdot \vec{u}=0
$$

A equação de momentum para fluidos Newtonianos incompressíveis é apresentada na Equação (2).

$$
\begin{aligned}
\frac{\partial}{\partial t}(\rho \vec{u})+\nabla \cdot(\rho \vec{u} \vec{u}) & \\
& =-\nabla P+\nabla \cdot\left\{\mu\left[\nabla \vec{u}+(\nabla \vec{u})^{T}\right]\right\}+\rho \vec{g} \\
& +F_{S}
\end{aligned}
$$

A força de tensão superficial na interface gás-líquido é expressa de acordo com a Equação (3).

$$
F_{S}=\frac{\sigma\left(\rho \kappa \nabla \alpha_{l}\right)}{0,5\left(\rho_{l}+\rho_{g}\right)}
$$

Sendo:

$$
\begin{aligned}
& \text { к }=\nabla \cdot \hat{n} \\
& \hat{n}=\frac{n}{|n|}
\end{aligned}
$$$$
n=\nabla \alpha_{k}
$$

Onde $\sigma$ é o coeficiente de tensão superficial, $\hat{n}$ é a superfície unitária do vetor normal, o qual é estimado a partir do gradiente da fração volumétrica $\mathrm{e} \kappa$ é a curvatura da superfície local. O subscrito $l$ e $g$ representam a fase líquida e gasosa, respectivamente.

No modelo VOF, o rastreamento da (s) interface (s) entre as fases é realizado pela solução da equação de continuidade para a fração volumétrica de uma ou várias fases. Para uma fase $k$, a equação da fração volumétrica é dada pela Equação (7).

$$
\frac{\partial}{\partial t} \alpha_{k}+\vec{u}_{k} \cdot \nabla \alpha_{k}=0
$$

O cálculo da fração volumétrica da fase primária é baseado na restrição (8):

$$
\sum_{k=1}^{2} \alpha_{k}=1
$$

Para obter uma representação precisa e clara da interface gás-líquido, adota-se um plano de reconstrução geométrica baseado na abordagem linear por partes. No caso de um sistema binário gás-líquido, a densidade e a viscosidade da mistura de fluidos em uma célula computacional são obtidas 
pelas Equações (9) e (10), respectivamente.

$$
\begin{aligned}
& \rho(\vec{x}, t)=\alpha(\vec{x}, t) \rho_{l}+(1-\alpha(\vec{x}, t)) \rho_{g} \\
& \mu(\vec{x}, t)=\alpha(\vec{x}, t) \mu_{l}+(1-\alpha(\vec{x}, t)) \mu_{g}
\end{aligned}
$$

\subsection{Malha Computacional e Procedimento Numérico}

O domínio computacional foi baseado no aparato experimental exposto na Figura 1, o qual é mostrado na Figura 2.

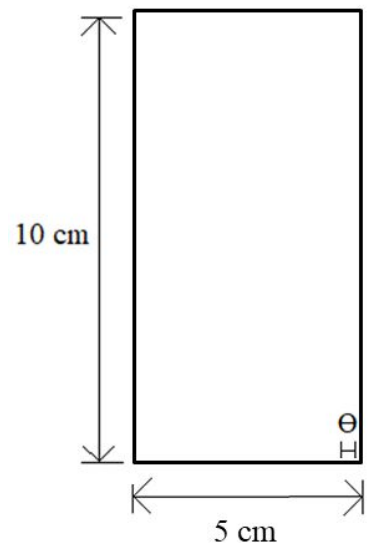

Figura 2 - Dimensões do domínio computacional.

A dimensão $\Theta$ refere-se ao diâmetro do orifício de injeção de ar. Devido ao elevado número de células geradas com o diâmetro real de orifício de entrada de $0,2 \mathrm{~mm}$, empregaram-se duas malhas computacionais com diâmetros diferentes, a fim de verificar-se seu comportamento sobre a distribuição e característica das bolhas. A geometria e a malha computacional foram geradas utilizando-se o software Gambit 2.4.6, considerando simetria em relação ao eixo y. A malha M1 possui $\Theta$ igual a $1 \mathrm{~mm}$, enquanto que a malha $\mathrm{M}-2$ apresenta $\Theta$ igual a $0,5 \mathrm{~mm}$. As características de cada malha são mostradas na Tabela 1 e as malhas computacionais são apresentadas na Figura 3.

Tabela 1 - Características das malhas computacionais.

\begin{tabular}{|cccc|}
\hline Malha & $\begin{array}{c}\text { Espaçamento } \\
\text { entre nós }(\mathrm{m})\end{array}$ & $\begin{array}{c}\mathrm{N}^{\circ} \text { de } \\
\text { células }\end{array}$ & Qualidade \\
\hline M-1 & 0,0005 & 20.000 & 1 \\
M-2 & 0,00025 & 80.000 & 1 \\
\hline
\end{tabular}

a)

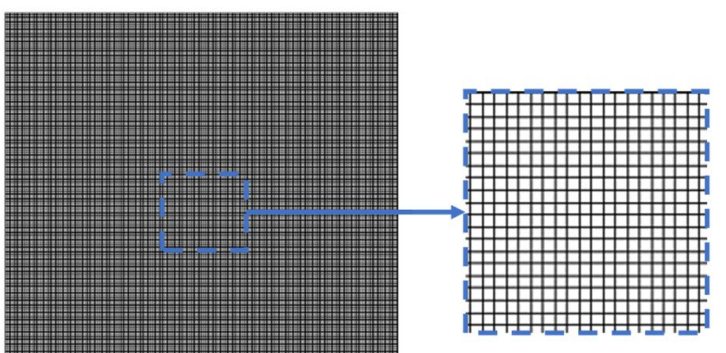

b)

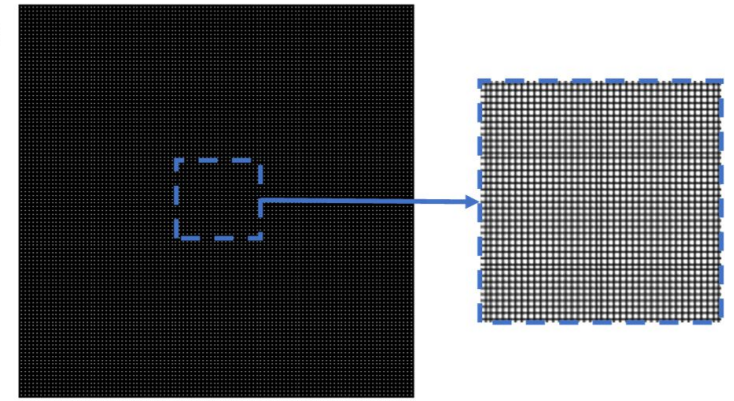

Figura 3 - Malhas computacionais: a) M-1; b) M-2.

A velocidade da injeção de ar foi calculada baseada na área de seção de entrada de cada malha, de forma a manter-se a vazão volumétrica de ar igual a $27,1 \mathrm{~mL} / \mathrm{min}$. O domínio foi preenchido com água (fase primária) e ar (fase secundária). No tempo $0,0 \mathrm{~s}$, injetou-se ar através do orifício de entrada. A velocidade de injeção de ar para a malha com orifício de diâmetro de $1 \mathrm{~mm}$ foi de $0,575 \mathrm{~m} / \mathrm{s}$, enquanto que para a de 0,5 $\mathrm{mm}$ foi de $2,3 \mathrm{~m} / \mathrm{s}$.

O tempo real de simulação para cada malha, variandose a tensão superficial, foi de $1 \mathrm{~s}$. Para inferir a velocidade das bolhas em cada simulação, ferramentas de pós-processamento disponíveis no software FLUENT foram empregadas, possibilitando a obtenção da velocidade média de bolhas na região caracterizada. Para medida do diâmetro de bolhas, para cada tensão superficial analisada, utilizou-se o software Image Pro Plus 6. Por meio do contorno de fração volumétrica de ar, calibrou-se no software a dimensão já conhecida do equipamento simulado $(10 \mathrm{~cm})$. Efetuou-se uma média aritmética entre comprimentos horizontal, vertical e de ambas diagonais de uma bolha. A média de tais comprimentos caracterizou o diâmetro da bolha de ar naquela condição.

A Tabela 2 apresenta os modelos e parâmetros da solução numérica empregados nas simulações do estudo fluidodinâmico da distribuição de bolhas de ar em um tanque de flotação utilizando-se diferentes tensões superficiais.

Tabela 2 - Modelos e parâmetros da solução numérica

\begin{tabular}{|c|c|}
\hline \multirow{3}{*}{ Condições de Contorno } & $\begin{array}{l}\text { Entrada do sistema: velocidade do } \\
\text { ar prescrita } \\
\text { Posição de saída: pressão } \\
\text { atmosférica }\end{array}$ \\
\hline & $\begin{array}{l}\text { Parede: não deslizamento e } \\
\text { impermeáveis }\end{array}$ \\
\hline & $\begin{array}{l}\text { Eixo de axissimetria foi } \\
\text { incorporado ao sistema. }\end{array}$ \\
\hline \multirow{4}{*}{ Método de Solução } & $\begin{array}{l}\text { Esquema de acoplamento pressão- } \\
\text { velocidade: PISO. }\end{array}$ \\
\hline & $\begin{array}{l}\text { Interpolação para equação do } \\
\text { momentum: QUICK }\end{array}$ \\
\hline & $\begin{array}{l}\text { Formulação Transiente: Implícita } \\
\text { de } 1^{\text {a }} \text { ordem. }\end{array}$ \\
\hline & $\begin{array}{l}\text { Interpolação de fração volumétrica: } \\
\text { Geo-Reconstruct }\end{array}$ \\
\hline $\begin{array}{l}\text { Critério de } \\
\text { Convergência }\end{array}$ & $\begin{array}{l}\text { Equação continuidade } \\
\text { movimento: } 1 \times 10^{-4} \text {. }\end{array}$ \\
\hline Passo no tempo & 0,0001 \\
\hline $\begin{array}{l}\text { Número de passos no } \\
\text { tempo }\end{array}$ & 10.000 \\
\hline
\end{tabular}
adotados nas simulações. 
$\mathrm{N}^{\circ}$ máximo de iterações

por passo

Parâmetro de relaxação

\section{RESULTADOS E DISCUSSÃO}

Em relação à malha $\mathrm{M}-1$, a Tabela 3 mostra as velocidades de bolhas de ar simuladas e medidas experimentalmente em função da tensão superficial, bem como o desvio em relação à medida experimental.

Tabela 3 - Velocidades das bolhas de ar simuladas para a malha M-1e medidas experimentalmente em função da tensão superficial.

$\begin{array}{cccc}\begin{array}{c}\text { Tensão } \\ (\mathbf{N} / \mathbf{m})\end{array} & \begin{array}{c}\text { Velocidade } \\ \text { Experimental } \\ (\mathbf{m} / \mathbf{s})\end{array} & \begin{array}{c}\text { Velocidade } \\ \text { Simulada } \\ (\mathbf{m} / \mathbf{s})\end{array} & \begin{array}{c}\text { Desvio } \\ (\mathbf{\%})\end{array} \\ 0,082 & 0,264 & 0,273 & 3,45 \\ 0,058 & 0,239 & 0,280 & 17,13 \\ 0,056 & 0,220 & 0,270 & 22,50 \\ 0,055 & 0,246 & 0,257 & 4,47 \\ 0,053 & 0,239 & 0,263 & 10,24 \\ 0,049 & 0,238 & 0,273 & 14,81\end{array}$

Em relação à malha $\mathrm{M}-2$, a Tabela 4 mostra as velocidades de bolhas de ar simuladas e medidas experimentalmente em função da tensão superficial, bem como o desvio em relação à medida experimental.

Tabela 4 - Velocidades das bolhas de ar simuladas para a malha M-2 e medidas experimentalmente em função da tensão superficial.

\begin{tabular}{cccc}
$\begin{array}{c}\text { Tensão } \\
(\mathbf{N} / \mathbf{m})\end{array}$ & $\begin{array}{c}\text { Velocidade } \\
\text { Experimental } \\
(\mathbf{m} / \mathbf{s})\end{array}$ & $\begin{array}{c}\text { Velocidade } \\
\text { Simulada } \\
(\mathbf{m} / \mathbf{s})\end{array}$ & $\begin{array}{c}\text { Desvio } \\
\mathbf{( \% )}\end{array}$ \\
\hline 0,082 & 0,264 & 0,297 & 12,28 \\
0,058 & 0,239 & 0,273 & 14,34 \\
0,056 & 0,220 & 0,287 & 30,07 \\
0,055 & 0,246 & 0,257 & 4,47 \\
0,053 & 0,239 & 0,297 & 24,20 \\
0,049 & 0,238 & 0,277 & 16,21 \\
\hline
\end{tabular}

Em relação às velocidades de ascensão das bolhas experimentais, não se obteve regularidade nos resultados, dado que essa grandeza apresentou comportamento oscilatório à medida que mais etanol foi adicionado. Isso pode ter sido causado pela dificuldade e imprecisão na medição desse fator. Resultado semelhante foi obtido para as simulações empregando as malhas M-1 e M-2.

A Figura 4 apresenta uma comparação entre os diâmetros experimentais e simulados em função da tensão superficial do líquido para as malhas M-1 e M-2.

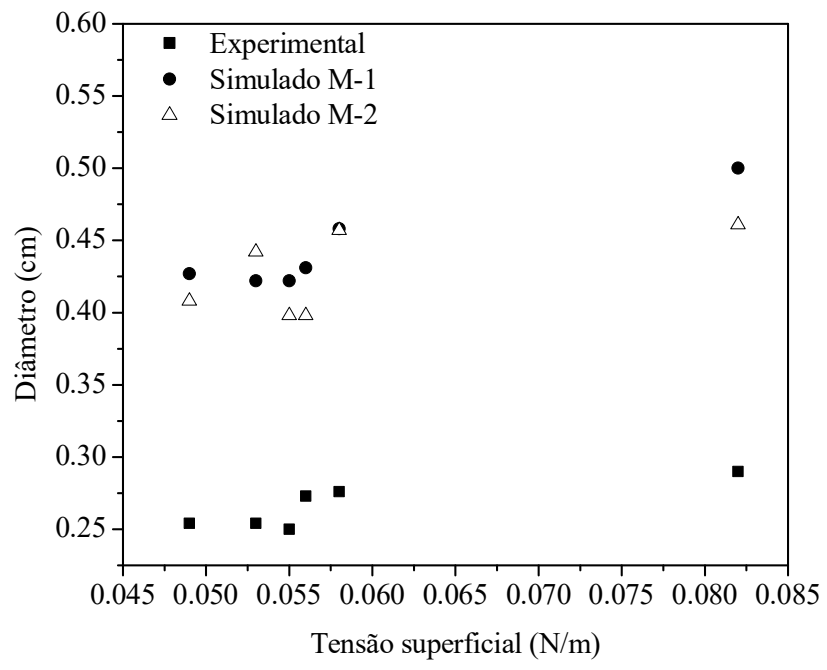

Figura 4 - Diâmetros das bolhas de ar simuladas para as malhas M-1 e M-2 e medidas experimentalmente em função da tensão superficial.

Em relação ao diâmetro de bolhas obtido experimentalmente, ao analisar a Figura 4, nota-se seu decréscimo à medida que o teor de etanol aumenta. Ou seja, a redução da tensão superficial do meio (provocada pela adição de etanol) facilita a maior coleta de partículas suspensas com base no mesmo volume de ar, visto que bolhas com diâmetros diminutos possuem grande área superficial. Logo, o processo de flotação torna-se mais eficaz.

Para a malha $\mathrm{M}-1$, as velocidades das bolhas simuladas apresentaram desvio máximo de $22,50 \%$ quando comparadas às experimentais. Em relação ao diâmetro de bolhas, os desvios apresentados em relação aos dados experimentais foram maiores (máximo de 72,41\%). O diâmetro das bolhas simuladas foi maior do que as experimentais. Esta diferença de tamanhos é justificada pela dificuldade encontrada para a realização das simulações, a partir da geração de uma malha computacional que tivesse diâmetro de orifício de entrada de ar igual ao experimental. Enquanto o diâmetro de injeção de ar experimental é $0,2 \mathrm{~mm}$, o diâmetro de injeção da malha computacional é de $1 \mathrm{~mm}$ para a malha M-1.

Ressalta-se que, quanto menor for o diâmetro de injeção de ar da malha computacional, mais refinada esta será, ocasionando maior esforço computacional, consequentemente, mais tempo para a solução das equações da continuidade e momentum. Com o intuito de analisar a influência do diâmetro de injeção de ar sobre as grandezas de velocidade e diâmetro de bolhas, construiu-se a malha M-2. A geração de uma malha com diâmetro de entrada de ar 50\% menor acarretou no aumento de 60.000 células em relação à malha M-1. Para a malha $\mathrm{M}-2$, a qual demandou consideravelmente mais tempo para a solução das equações do modelo, o desvio máximo obtido para as velocidades simuladas foi de $30,07 \%$ em relação às experimentais. Apesar de este valor ser maior do que o obtido com a malha $\mathrm{M}-1$, ainda representa baixa diferença quando comparado aos dados experimentais. No quesito de diâmetro de bolhas, o desvio máximo calculado foi de 74,13\% em relação aos valores experimentais. Este valor é quase o mesmo que o obtido utilizando a malha M-1. Para a situação analisada, a geração de uma malha computacional mais refinada, com metade do diâmetro do orifício de injeção de ar, 
não é justificável, visto que os desvios máximos de velocidade e diâmetro foram maiores, assim como o esforço computacional para resolução do modelo VOF.

Os perfis de fração de ar e velocidade de bolhas quando submetidas à tensão superficial $0,055 \mathrm{~N} / \mathrm{m}$ para a malha $\mathrm{M}-1$ e malha M-2, são apresentados nas Figuras 5 e 6 , respectivamente.
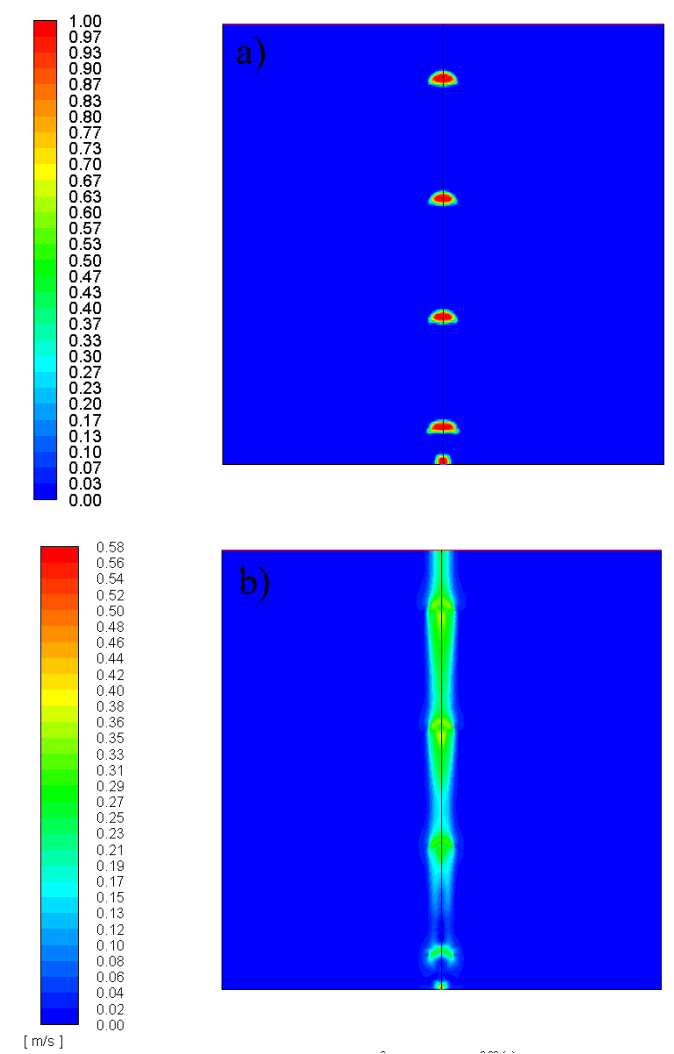

Figura 5 - a) Fração volumétrica de ar; b) velocidade de bolhas submetidos à tensão superficial de $0,055 \mathrm{~N} / \mathrm{m}$ para $a$ malha computacional M-1.
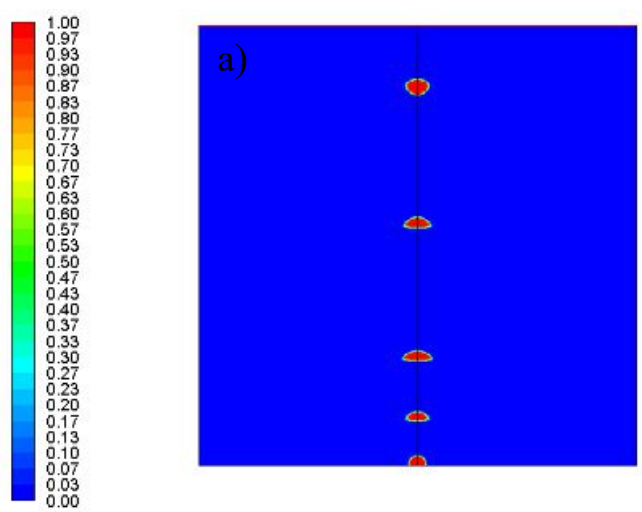
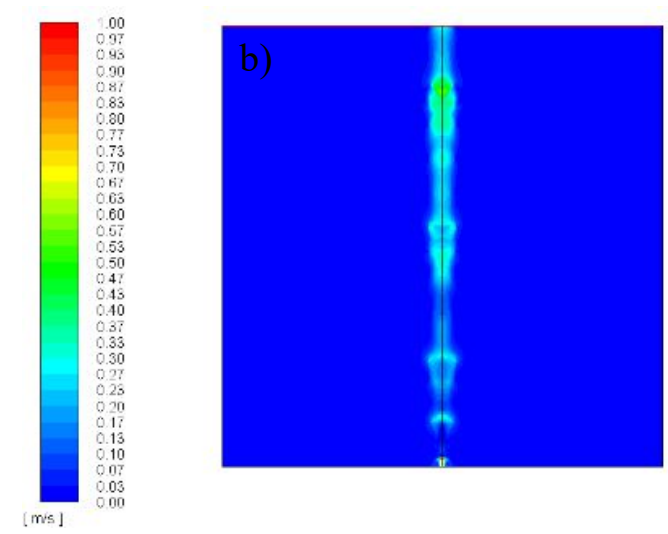

Figura 6 - a) Fração volumétrica de ar; b) velocidade de bolhas submetidos à tensão superficial de $0,055 \mathrm{~N} / \mathrm{m}$ para a malha computacional M-2.

A partir dos contornos de fração volumétrica de ar mostrados nas Figuras 5 e 6, observa-se no processo de formação da bolha a presença de três etapas: expansão, alongamento e distanciamento. Verifica-se que antes do desprendimento, a bolha cresce na direção vertical.

Durante as simulações foi observado o fenômeno de coalescência das bolhas de ar formadas, conforme mostrado na Figura 7. Através de uma sequência de contornos instantâneos de fração volumétrica de ar é possível verificar a coalescência entre duas bolhas sucessivas e uma rápida expansão da bolha formada na direção horizontal e vertical. Este comportamento também foi observado experimentalmente. Este fenômeno indica que as simulações representaram bem o sistema estudado.

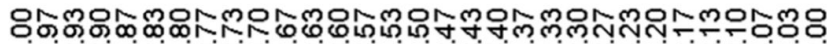
$-000000000000000000000000000000$
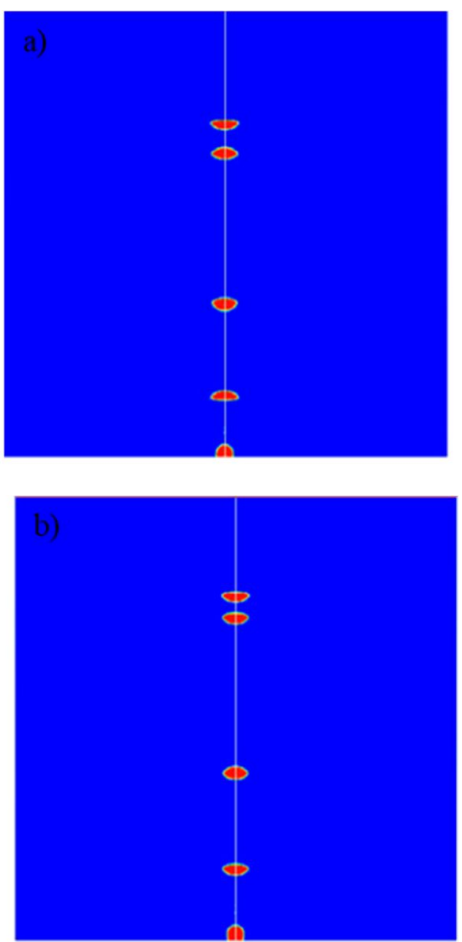

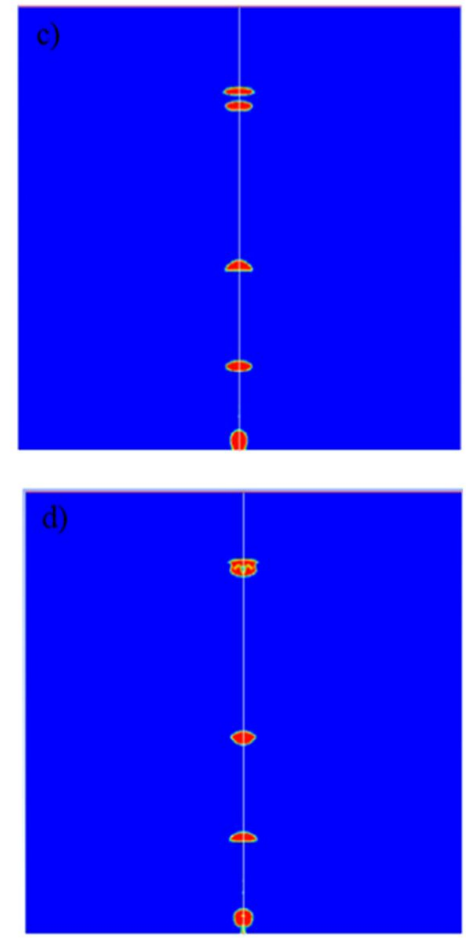

Figura 7 -Coalescência de bolha de ar.

\section{CONCLUSÕES}

A adição do tensoativo etanol em água causou diminuição da tensão superficial do líquido e esta diminuiu conforme aumentou a concentração de etanol. À medida que aumentou o teor do tensoativo nas cubetas, menores tamanhos de bolhas foram obtidos, favorecendo o processo de flotação.

Tanto para a malha M-1 quanto M-2, os desvios entre as velocidades das bolhas de ar simuladas em relação às experimentais foram aceitáveis, correspondendo a valores máximos de $22,50 \%$ e $30,07 \%$, respectivamente. Quanto ao diâmetro das bolhas de ar, as simulações apresentaram diâmetros de bolha maiores que os experimentais, com desvios máximos de $72,41 \%$ e $74,13 \%$ para as malhas $M-1$ e $\mathrm{M}-2$, respectivamente. $\mathrm{O}$ perfil de diâmetro de bolhas em função das tensões superficiais seguiu a mesma tendência dos resultados experimentais. Para o sistema estudado e simulações realizadas, deve-se optar pela malha computacional M-1, visto que os desvios máximos de velocidade e diâmetro de bolhas foram menores, assim como o esforço computacional para solução das equações da continuidade e momentum do modelo VOF. No entanto, é necessário realizar maiores investigações acerca do modelo e procedimento experimental empregado para justificar o motivo de a malha mais refinada (M2) apresentar maiores desvios.

\section{AGRADECIMENTOS}

Os autores agradecem o apoio financeiro concedido pela Coordenação de Aperfeiçoamento de Pessoal de Nível Superior - Brasil (CAPES) - Código de Financiamento 001 e pelo Conselho Nacional de Desenvolvimento Científico e Tecnológico - Brasil (CNPq).

\section{R E F E RÊNCIAS}

ABBASSI, W.; BESBES, S.; EL HAJEM, M.; BEN AISSIA, H.; CHAMPAGNE, J. Y.; \& JAY, J. Influence of operating conditions and liquid phase viscosity with volume of fluid method on bubble formation process. European Journal of Mechanics B/Fluids, v. 65, p. 284 298, 2017.

ABBASSI, W.; BESBES, S.; ELHAJEM, M.; AISSIA, H. B.; CHAMPAGNE, J. Y. Numerical simulation of free ascension and coaxial coalescence of air bubbles using the volume of fluid method (VOF). Computers \& Fluids, v. 161, p. 47-59, 2018.

BEHRING J. L.; LUCAS, M.; MACHADO C.; BARCELLOS I. O. Adaptação no método do peso da gota para determinação da tensão superficial: um método simplificado para a quantificação da CMC de surfactantes no ensino da química. Química Nova, v. 27, n. 3, p. 492-495, 2004.

PENNA, R.; OLIVEIRA, M. L. M.; VALADÃO, G. E. S.; PERES, A. E. C. Estudo comparativo entre dois sistemas de aeração de coluna de flotação. Revista Escola de Minas, v. 56, n. 3, p.195-200, 2003.

REIS FILHO, A. M.; GARDUSI, F.; LUZ, P.H.M.; CARVALHO, G.R.L.; REIS, A.S.; BARROZO, M.A.S. Avaliação da distribuição do diâmetro de bolhas em coluna de flotação. XIX Jornada de Engenharia Química, UFU, Uberlândia (MG), 2014.

RODRIGUES, R. T. Desenvolvimento da técnica LTM-BSizer para a caracterização de bolhas e avaliação de parâmetros no processo de flotação. 2004. 114 f. Tese (Doutorado em Engenharia de Minas), UFRGS, Porto Alegre, RS, 2004.

RODRIGUES, J. P.; BÉTTEGA, R. Evaluation of multiphase CFD models for Dissolved Air Flotation (DAF) process. Colloids and Surfaces A: Physicochemical and Engineering Aspects, v. 539, p. 116-123, 2018.

RODRIGUES, J. P.; BATISTA, J. N.M.; BÉTTEGA, R. Application of population balance equations and interaction models in CFD simulation of the bubble distribution in dissolved air flotation. Colloids and Surfaces A: Physicochemical and Engineering Aspects, v. 577, p. 723-732, 2019.

SHAWN D. J. Introdução à química dos coloides e de superfícies. São Paulo: Editora Blucher, 1975. 42 p. 